\title{
Measurement of Growth Hormone-releasing Hormone and Somatostatin in Hypothalamic-Portal Plasma of Unanesthetized Sheep \\ Spontaneous Secretion and Response to Insulin-induced Hypoglycemia
}

Lawrence A. Frohman, Thomas R. Downs, lain J. Clarke, and Gregory B. Thomas

Division of Endocrinology and Metabolism, Department of Medicine, University of Cincinnati College of Medicine, Cincinnati, Ohio 45267; and Medical Research Center, Prince Henry Hospital, Melbourne, Australia

\begin{abstract}
To elucidate the role of growth hormone (GH)-releasing hormone (GRH) and somatostatin (SRIH) in the regulation of the growth hormone (GH) secretory pattern, we collected portal blood from five unanesthetized ovariectomized ewes for repeated measurements of GRH and SRIH simultaneous with those of peripheral GH. Hormones were measured at 10-min intervals for $5.5 \mathrm{~h}$ and their interrelationships analyzed. Mean portal GRH was $20.4 \pm 6.7$ (SD) $\mathrm{pg} / \mathrm{ml}$ and the estimated overall secretion rate was $13 \mathrm{pg} / \mathrm{min}$. GRH secretion was pulsatile with peaks of $25-40 \mathrm{pg} / \mathrm{ml}$ and a mean pulse interval of 71 min. Mean portal SRIH was $72 \pm 33 \mathrm{pg} / \mathrm{ml}$ and the estimated overall secretion rate was $32 \mathrm{pg} / \mathrm{min}$. SRIH secretion was also pulsatile with peaks of $65-160 \mathrm{pg} / \mathrm{ml}$ and a mean pulse interval of $54 \mathrm{~min}$. The GH pulse interval was $62 \mathrm{~min}$. A significant association was present between GRH and GH secretory peaks though not between GRH and SRIH or SRIH and GH. Insulin hypoglycemia resulted in a rapid and brief stimulation of SRIH secretion followed by a decline in GH levels. No effect was observed on GRH secretion until $90 \mathrm{~min}$, when a slight increase occurred. The results suggest $(a)$ the presence of an independent neural rhythmicity of GRH and SRIH secretion with a primary role of GRH in determining pulsatile GRH secretion, and (b) that the inhibitory effects of insulin hypoglycemia on GH in this species are attributable to a combination of enhanced SRIH secretion and possibly other factors, though without significant inhibition of GRH. (J. Clin. Invest. 1990. 86:17-24.) Key words: growth hormone-releasing hormone • somatostatin • growth hormone $\cdot$ hypothalamic-portal $\bullet$ sheep
\end{abstract}

\section{Introduction}

Growth hormone $(\mathrm{GH})^{1}$ secretion occurs in an episodic manner characterized by pulses of relatively short duration interspersed with troughs during which time circulating GH levels

Address correspondence and reprint requests to Lawrence A. Frohman, M.D., Division of Endocrinology and Metabolism, University of Cincinnati Medical Center, 231 Bethesda Avenue, ML \#547, Cincinnati, OH 45267.

Received for publication 5 September 1989 and in revised form 24 January 1990.

1. Abbreviations used in this paper: $\mathrm{CV}$, coefficient of variation; $\mathrm{GH}$, growth hormone; GRH, GH-releasing hormone; hGRH, human GRH; IR, immunoreactive; oGRH, ovine GRH; SRIH, somatostatin; TFA, trifluoroacetic acid.

J. Clin. Invest.

(c) The American Society for Clinical Investigation, Inc.

0021-9738/90/07/0017/08 \$2.00

Volume 86, July 1990, 17-24 approach the sensitivity of detection by current techniques (1). The neuroendocrine mechanism that regulates $\mathrm{GH}$ secretion is mediated by two hypothalamic hormones, one of which is stimulatory (GH-releasing hormone [GRH]) and the other, inhibitory (somatostatin [SRIH]). Although their effects on GH secretion have been studied extensively, there have been formidable technical difficulties in obtaining simultaneous measurements of the two neurohormones in hypothalamichypophysial portal blood and comparing their secretory patterns with those of $\mathrm{GH}$ in peripheral blood under physiologic conditions. Thus, the pattern of their release from the median eminence into the hypothalamic-hypophysial portal system and current knowledge of their precise interaction in regulating pulsatile GH secretion is based almost entirely on indirect evidence. Initial results of passive immunization with anti-GRH serum (2) and anti-SRIH serum (3) suggested that pulsatile GH secretion was dependent on pulses of GRH and that the role of SRIH was primarily to set the level of the GH responses to GRH. However, subsequent studies with infusions of anti-SRIH serum (4), comparison of $\mathrm{GH}$ responses to GRH during trough and pulse periods of GH secretion (5), and interruption of intrahypothalamic somatostatinergic neuronal circuitry (6) suggested the presence of intermittent and possibly pulsatile secretion of SRIH as well as GRH.

Attempts to measure GRH and SRIH in peripheral circulation have not clarified their hypophysiotropic roles since $(a)$ SRIH immunoreactivity is believed attributable to gastro-pancreatic rather than hypothalamic origin (7), and (b) GRH immunoreactivity remains to be critically characterized and may also be of nonhypothalamic origin (8). One study has reported repeated measurements of immunoreactive GRH and SRIH in portal blood of rats and demonstrated a slight decrease in SRIH levels during a 20-min period before a rise in GRH levels was observed (9). However, the use of anesthetized animals and the necessity of hypophysectomy in order to collect portal blood limited the overall interpretation of these results.

Recently, a technique has become available for repeated sampling of hypothalamic-hypophysial portal blood in unanesthetized sheep and has been used successfully in demonstrating a high degree of concordance between pulsatile secretion of gonadotropin-releasing hormone and luteinizing hormone $(10,11)$. In the present report, we describe the use of this model to examine the interaction of hypothalamic GRH and SRIH secretion in the regulation of GH under basal conditions and in response to insulin-induced hypoglycemia.

\section{Methods}

\section{Animals and experimental techniques}

Studies were performed in five mature Corriedale ewes (38-44 kg) that had been ovariectomized for at least 3 mo before portal blood collection. During experiments, the sheep were housed in individual pens under natural photoperiodic conditions and given a maintenance ra- 
tion of lucerne chaff and grain with free access to water. The sheep were prepared for portal blood collection as previously described (10, 11). Briefly, under halothane anesthesia, a tunnel was drilled into the sphenoid bone from the back of the nasal cavity to create an artificial sinus immediately anterior to the pituitary gland. Two $12-\mathrm{G}$ needles were implanted through the nasal bone so that one was directed at the portal vessels on the face of the gland, and the other opened into the floor of the artificial sinus. The artificial sinus was then sealed off from the nose with dental acrylic and the animal allowed to recover. $24 \mathrm{~h}$ later, a catheter was placed in the external jugular vein for collection of peripheral blood and the animal was heparinized. A 16-G stilette was then passed through the upper needle and used to puncture the portal veins to a depth of 2-3 mm on the anterior surface of the pituitary. Based on previous experience, $\sim 50 \%$ of the portal vessels are transected by this procedure. Extreme care is taken when positioning the needles to avoid cutting too far laterally and damaging the cavernous sinus. The needle position and extent of transection were verified at autopsy in each animal. Portal blood was withdrawn from the artificial sinus by constant suction applied to a catheter in the lower needle. Blood was collected at 10 - (or occasionally 5-) min intervals into tubes containing aprotinin $(500 \mathrm{KJU} / \mathrm{ml}$ blood) and immediately chilled. The volume of each portal blood sample was $\sim 3.0 \mathrm{ml}$ and was fairly constant, varying by only $\sim 20 \%$ throughout the experimental period. Bacitracin, used as a peptidase inhibitor in previous experiments (10), was omitted after preliminary studies indicated interference in the GRH RIA. A sample of jugular blood $(5.0 \mathrm{ml})$ was obtained every 5 or $10 \mathrm{~min}$, immediately after the end of each portal collection period. On the basis of previous studies (12), the contribution of retrograde pituitary blood flow to the diverted portal blood is believed to be minimal. Observation of the animals did not indicate any evidence of stress during the sampling period except that due to handling at the beginning of the experiment.

After a 5-h blood sampling period, regular insulin (Commonwealth Serum Laboratories, Melbourne; $5 \mathrm{U} / \mathrm{kg}$ ) was injected intravenously and portal and peripheral blood samples collected for an additional 2 h. Previous studies have shown this dose of insulin to reliably lower the mean plasma glucose levels within $40 \mathrm{~min}$ to $\sim 20 \mathrm{mg} / \mathrm{dl}$ (13). Total blood removal during the entire study was $\sim 320 \mathrm{ml}$, which represented $8 \%$ of blood volume. Hematocrit determinations, performed at 2-3-h intervals, revealed no significant change during the study period. Blood samples were centrifuged at $4^{\circ} \mathrm{C}$ and the plasma stored at $-20^{\circ} \mathrm{C}$ until transported from Melbourne to Cincinnati on dry ice.

\section{Plasma extraction and hormone assays}

In preliminary experiments, ovine plasma was acidified with $0.2 \mathrm{ml} 1.0$ $M$ trifluoroacetic acid (TFA)/ml plasma and extracted on a reverse phase $\mathrm{C}_{18}$ Sep-Pak (Waters Associates, Milford, MA) as previously described (14). Retained substances were eluted with $2 \mathrm{ml} 80 \%$ acetonitrile/20\% $0.01 \mathrm{M}$ TFA and the eluate was lyophilized. Initially, peptide recovery was evaluated by the addition of synthetic ${ }^{125} \mathrm{I}$-labeled peptides to ovine plasma and counting the eluate radioactivity. The recovery of added [ $\left.{ }^{125} \mathrm{I}\right] \mathrm{SRIH}$ and $\left[{ }^{125} \mathrm{I}\right]$ ovine (o)GRH was $>90 \%$ and $75-80 \%$, respectively $\left(\left[{ }^{125} I\right]\right.$ human (h)GRH recovery using identical conditions was $>90 \%$ ). The recovery of $\left[{ }^{125} \mathrm{I}\right] \mathrm{OGRH}$ could be slightly improved $(80-85 \%)$ by recycling the acidified plasma sample one additional time through the Sep-Pak and eluting with $90 \%$ acetonitrile/ $10 \% 0.01 \mathrm{M}$ TFA. The recovery of unlabeled peptides added to ovine plasma was next evaluated by RIA after resuspension of the lyophilisate in assay buffer $(0.05 \mathrm{M}$ sodium phosphate, $0.9 \% \mathrm{NaCl}, 0.5 \% \mathrm{BSA}$, $0.025 \mathrm{M}$ EDTA, $0.02 \%$ protamine sulfate, $0.01 \%$ Tween-20, $\mathrm{pH} 7.5$ ). SRIH recovery remained at $>90 \%$, while $h$ GRH and oGRH recovery decreased to $10-20 \%$ (hGRH and oGRH recovery from human plasma using identical conditions remained at $>90 \%$ and $80-85 \%$, respectively). The final recovery of $O G R H$ or hGRH from the ovine plasma extract was increased to a maximum of $65-70 \%$ by resuspending the lyophilisate in $5 \mathrm{mM}$ acetic acid, $0.25 \% \mathrm{BSA}, 0.01 \%$ Tween-20 (ABT).
A 1-ml aliquot of portal and jugular plasma from each animal at each time point was extracted as a group, together with three 1-ml aliquots of an ovine jugular plasma pool with added oGRH and SRIH to monitor recovery. The extracts were lyophilized, resuspended in $\mathbf{0 . 3}$ $\mathrm{ml} \mathrm{ABT}$, centrifuged $\left(10,000 \mathrm{~g}, 10 \mathrm{~min}, 4^{\circ} \mathrm{C}\right)$, and the supernatants assayed in duplicate for oGRH and SRIH (100- and 25- $\mu 1$ aliquots, respectively). The recovery of SRIH and $O$ GRH from the plasma pools was $95 \pm 2 \%$ and $66 \pm 2 \%$ (mean \pm SE, $n=5$ each), respectively. Results were not corrected for recovery.

Plasma oGRH was measured by a modification of the previously described RIA for hGRH (14), using anti-hGRH(1-40)-OH antiserum (No. 871), [ ${ }^{125}$ I]hGRH(1-40)-OH tracer, and an OGRH standard (Peninsula Laboratories, Inc., Belmont, CA). The crossreactivity of oGRH was $80 \%$ compared with the hGRH(1-40)-OH standard with parallel displacement. All portal and jugular samples from each animal were measured in a single assay. The intra- and interassay coefficients of variation (CVs) were 12.5 and $10.6 \%$, respectively, and the average sensitivity of the assay was $2.7 \mathrm{pg} /$ tube $(9 \mathrm{pg} / \mathrm{ml}$ plasma). Plasma SRIH was measured by RIA, as previously reported $(15,16)$, with portal and jugular samples from each animal measured in a single assay. The average sensitivity was $1.5 \mathrm{pg} /$ tube $(5 \mathrm{pg} / \mathrm{ml}$ plasma), with intra- and interassay CVs of 7.1 and $11.2 \%$, respectively. The dilution curves of immunoreactive (IR) $O$ GRH and SRIH in portal plasma were parallel to those of synthetic oGRH and hGRH and SRIH, respectively.

Ovine GH was measured by RIA using the reagents provided by the National Hormone Pituitary Program (Baltimore, MD). All jugular samples were measured in duplicate with an intraassay CV of $10.4 \%$. The average sensitivity was $25 \mathrm{pg} /$ tube $(500 \mathrm{pg} / \mathrm{ml}$ plasma) based on the National Institute of Arthritis, Diabetes, and Digestive and Kidney Diseases OGH-I-4 reference standard. For purposes of statistical analysis (see below) the least detectable value for the particular assay was assigned to all values that were at or beneath that level. Blood glucose was measured by a glucose oxidase method.

\section{Statistical analysis}

Comparison of spontaneous secretory patterns of GRH, SRIH, and $G H$. We initially determined whether directional changes in GRH (concordant) and/or SRIH (discordant) occurred immediately before (i.e. in the preceding sample) or simultaneous with changes in GH or the other hypothalamic hormone. A computer program was developed to tabulate the frequency of such changes between pairs of hormones in each individual animal during the 5-h period when spontaneous secretion was monitored. Multiple criteria for change were examined, ranging from 0 to $10 \mathrm{pg} / \mathrm{ml}$ (GRH or SRIH) and 0 to $3 \mathrm{ng} / \mathrm{ml}(\mathrm{GH})$ (absolute) and 0 to $200 \%$ (relative). The resulting frequencies (expressed as a percentage) in the five animals ("experimental") were then compared with that expected by random association and generated by comparing directional changes in hormone values from different animals ("random") rather than the same animal. Thus, four separate sets of random frequencies were available for each animal, giving a total of 20 random series with which to compare the results in the five individual animals. Differences between the two groups were determined by Student's $t$ test.

The pulses present in each hormone secretory profile during the 5-h spontaneous secretion period were determined by the program Detect (17) (method 1). The frequency with which pulses of GRH or SRIH occurred immediately preceding or concurrently with those of GH or of one another in individual animals was compared with the random frequency observed using pairs of animals as described above. Statistical differences in frequency were determined by the chi square test. As a validation of the appropriateness of the Detect algorithm, pulses were also determined, using as criteria, an increase of at least three times the CV greater than the preceding minimum trough value. The trough minimum was defined as the lowest value in a series of hormone measurements beginning with a decreasing value and including all subsequent values that $(a)$ were no greater than three times the $C V$ of the lowest included value and $(b)$ did not initiate a progressive rise that 
culminated in a level at least three times the CV of the lowest included value (method 2 ).

Insulin-induced hypoglycemia. Mean GRH, SRIH, and GH values after insulin administration were compared by a repeated measures analysis of variance. A $\boldsymbol{P}<\mathbf{0 . 0 5}$ was considered significant.

\section{Results}

Recovery of immunoreactive GRH from ovine portal plasma. The recovery of ${ }^{125}$ I-labeled oGRH from either ovine or human plasma was always consistently lower (10-15\%) than that of $\left[{ }^{125} \mathrm{I}\right] \mathrm{hGRH}$. This appeared to be primarily the result of a slightly reduced elution efficiency for oGRH from the SepPak cartridge and occurred in the presence or absence of plasma. The major problem encountered was with the recovery of GRH from lyophilized ovine plasma extracts. RIA of ovine plasma extracts containing added unlabeled oGRH or hGRH, after resuspension in RIA buffer, revealed that only $10-20 \%$ of the added peptide was measurable, while the recovery of these peptides from human plasma using identical conditions was the same as that seen with $\left[{ }^{125} \mathrm{I}\right]$ oGRH $(80-85 \%)$ and $\left[{ }^{125} \mathrm{I}\right] \mathrm{hGRH}(>90 \%)$. Resuspension of the lyophilized ovine plasma extract in an acidic solution $(\mathrm{pH}<5)$ or at low salt concentration increased the recovery of GRH from ovine portal or jugular plasma to a maximal level of $65-70 \%$. Whether this effect is due to the presence of a substance(s) with specific or nonspecific binding affinity for GRH is unknown. However, the same difficulties have been encountered with the recovery of GRH from bovine plasma extracts (data not shown), while GRH recovery from human, porcine, and murine plasma extracts by our standard protocol has been very efficient ( $>90 \%)$.

Jugular venous samples were also assayed for $O G R H$ and SRIH in all five sheep. Of a total of 135 samples, IR-oGRH was detected in 26 (19\%), though in all samples, values were $<30 \mathrm{pg} / \mathrm{ml}$ and generally less than double the least detectable value for the particular assay. These barely detectable values were insufficient for further characterization. SRIH was detected in $121(82 \%)$ of 147 jugular plasma samples. Values were almost always less than half of those in portal plasma and exhibited an overall mean \pm SD of $21 \pm 13 \mathrm{pg} / \mathrm{ml}$.

Spontaneous secretory pattern of portal GRH and SRIH and of peripheral GH. The secretory patterns of GRH and SRIH in portal plasma and of GH in jugular plasma during the 5-h observation period for each of the animals are shown in Fig. 1. IR-GRH was detectable in all of the samples from three sheep and in 87 and 58\% of samples from the other two animals. Overall mean portal GRH was $20.4 \pm 6.7 \mathrm{pg} / \mathrm{ml}$ (mean $\pm \mathrm{SD}$ ). On the basis of a $0.3-\mathrm{ml} / \mathrm{min}$ blood collection, a hematocrit of $29.0 \pm 2.2 \%$, an extraction efficiency of $66 \%$, and assuming a $50 \%$ removal of portal blood by the surgical technique (resulting in a corrected portal blood flow of $0.6 \mathrm{ml} /$ $\mathrm{min}$ ), the mean hypothalamic secretory rate of GRH was calculated to be $13.2 \mathrm{pg} / \mathrm{min}$.

GRH secretion was pulsatile in each of the animals with peak values ranging from 25 to $40 \mathrm{pg} / \mathrm{ml}$. The number of pulses detected during the 5 -h period (method 1 ) ranged from 3 to 6 with a mean of $4.2 \pm 1.1$, representing a mean pulse interval of $71 \mathrm{~min}$. An identical number of pulses (4.2 \pm 1.3 ) were detected using method 2.

SRIH was detectable in all of the portal samples from each of the sheep. Overall mean portal SRIH was $72 \pm 33 \mathrm{pg} / \mathrm{ml}$ and the mean hypothalamic secretory rate of SRIH was calculated (as above) to be $32.3 \mathrm{pg} / \mathrm{min}$. SRIH secretion was pulsatile in each of the animals, with peak values ranging from 65 to 160 $\mathrm{pg} / \mathrm{ml}$. The number of pulses detected during the 5-h period (method 1) ranged from 4 to 9 with a mean of 5.6 \pm 2.1 , representing a mean pulse interval of $54 \mathrm{~min}$. Using method 2 , $5.8 \pm 1.9$ pulses were detected, representing a mean pulse interval of $52 \mathrm{~min}$.

Plasma GH levels were detectable in $97 \%$ of the jugular samples. Overall mean jugular plasma $\mathrm{GH}$ was $2.9 \pm 2.2 \mathrm{ng} / \mathrm{ml}$. GH secretion exhibited pulsatility in each of the sheep, with peak values ranging from 4 to $11 \mathrm{ng} / \mathrm{ml}$. Using method 1 , the mean number of $\mathrm{GH}$ pulses during the 5 -h period was 4.6 \pm 2.2 , representing a mean pulse interval of $65 \mathrm{~min}$, whereas with method 2 , the values were $5.2 \pm 1.6$ pulses and $58 \mathrm{~min}$, respectively.

Relationships among GRH, SRIH, and GH levels. Using the algorithm described for comparing directional changes in GRH, SRIH, and GH levels, we found that a substantial percentage of concordance in directional changes between GRH and GH and discordance in directional changes between SRIH and GH when examined at simultaneous time periods or with a one sample lag time for the GH value (Fig. 2). However, when these percentages were compared with those that would be expected randomly (by analyzing hormone values from different animals), there were no significant differences. The curves of the random comparisons were virtually superimposable on those derived from the intra-animal comparisons in the five sheep. Subsequent analyses therefore used GRH, SRIH, and GH peaks and SRIH troughs as determined by methods 1 and 2 .

Using hormone peaks as defined by method 1, we determined the percentage of $\mathrm{GH}$ peaks occurring coincident with or immediately after (one sample) those of GRH or SRIH and also the interrelationship of GRH and SRIH peaks. The results are shown in Table I. 38\% of the GH peaks occurred coincident with GRH peaks and $62 \%$ occurred either coincident with or immediately (one sample later) after GRH peaks. These associations were statistically significant when compared with random associations from different animals $(P<0.05$ and $P$ $<0.02$, respectively) or to associations with SRIH peaks ( $P$ $<0.02$ and $P<0.01$, respectively). In contrast, GH peaks coincident with SRIH peaks were not significantly different between experimental and random pairings. Similarly, the frequency of GRH peaks immediately preceding SRIH peaks or SRIH peaks immediately preceding GRH peaks did not differ significantly between experimental and random pairings.

The results of analyses using peaks defined by method 2 are shown in Table II. The association of GRH and GH peaks was qualitatively similar to that obtained with method 1 (data not shown). GRH peaks were found to occur coincident with (28\%, $P=0.025)$ or immediately preceding $(48 \%, P<0.01)$ the initiation of the GH peak. There was no association of GH peak initiation with SRIH troughs.

Effect of insulin-induced hypoglycemia on portal GRH and SRIH and jugular GH levels. The results of insulin-induced hypoglycemia are shown in Fig. 3. All animals developed profound hypoglycemia (initial plasma glucose [time 0]: $45 \pm 4$ $\mathrm{mg} / \mathrm{dl}$; nadir plasma glucose [time $40 \mathrm{~min}$ ]: $18 \pm 2 \mathrm{mg} / \mathrm{dl}$ ) which persisted for at least $90 \mathrm{~min}(18 \pm 1 \mathrm{mg} / \mathrm{dl})$. GRH values were unaltered by insulin-induced hypoglycemia until $90 \mathrm{~min}$ after injection, when a slight increase was observed. SRIH 

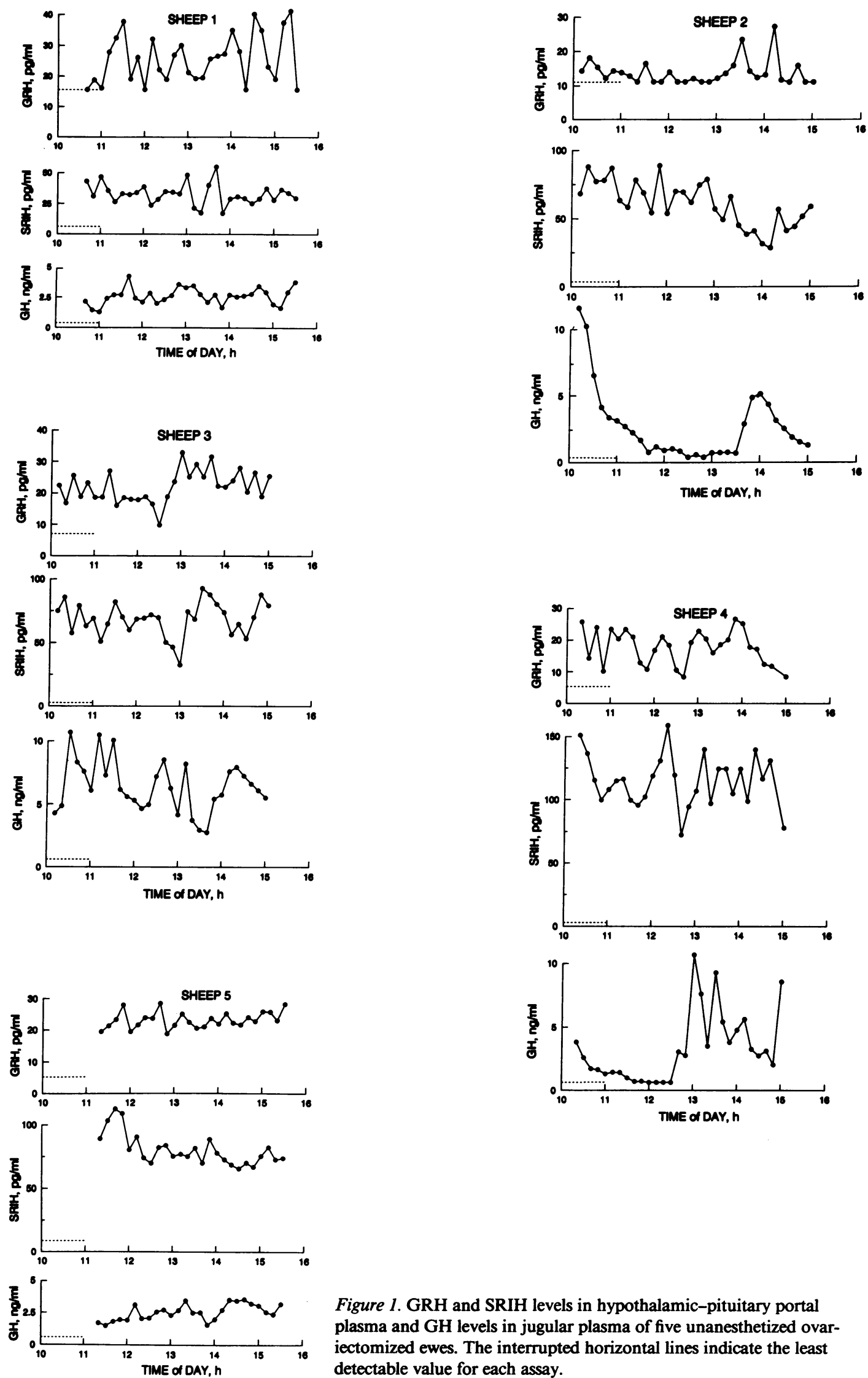

Figure 1. GRH and SRIH levels in hypothalamic-pituitary portal plasma and GH levels in jugular plasma of five unanesthetized ovariectomized ewes. The interrupted horizontal lines indicate the least detectable value for each assay. 

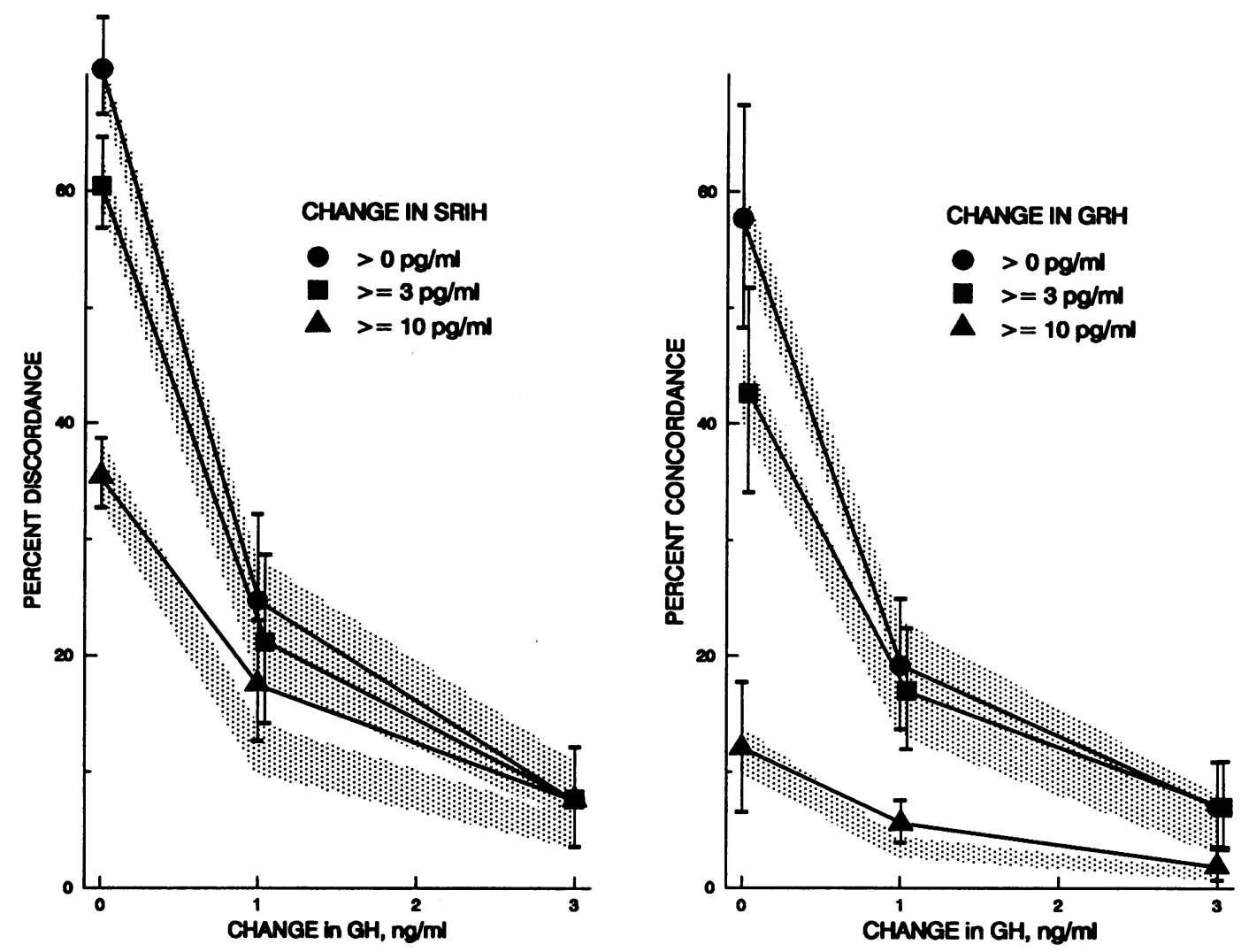

Figure 2. Frequency of discordance between directional changes in SRIH and GH levels and of concordance between directional changes in GRH and GH levels in individual sheep $(n=5)$, as compared with the expected frequency of concordance and discordance in values using data from different sheep $(n=20)$ at the indicated levels of change in each parameter. See Methods for details. The mean $\pm \mathrm{SE}$ of the results in individual animals are shown superimposed on a stippled area representing the mean $\pm S E$ of the results in different animals.

values, however, exhibited an increase of $93 \%(P<0.01) 20$ min after insulin injection that remained significantly elevated until $50 \mathrm{~min}$ after injection, at which time they returned to preinjection levels. GH values exhibited a gradual decline which was significant from 60 through $110 \mathrm{~min}$.

\section{Discussion}

The results of the present studies demonstrate that both GRH and SRIH can be reliably measured in nearly all portal samples obtained at 10-min intervals from unanesthetized sheep using the sampling technique described. Our model therefore permits the study of the GRH/SRIH/GH axis in unstressed animals. The sensitivity of the technique is limited only by the detection limits of each assay and the fraction of hypothalamic-pituitary portal blood that is diverted from the pituitary (18). The technique cannot exclude the possibility of admixture of portal blood with retrograde flow from the pituitary. Thus, the actual portal GRH levels may be slightly higher since measurements of GRH in jugular plasma revealed detectable values in $<20 \%$ of samples.

GRH secretion, as reflected in portal blood GRH levels, was observed to be pulsatile in all animals with a mean pulse interval of $71 \mathrm{~min}$. Two separate statistical methods were used to determine this value, one of which (Detect [17]) has been validated in studies using peripheral blood measurements and the other of which was developed specifically for these studies. The comparability of the pulse analyses by the two methods helps support the conclusion that the results are not technique dependent. The absence of any recirculation of GRH in the portal vascular system actually simplifies the interpretation of the results since hormone metabolic clearance (i.e., half life) need not be considered.

SRIH secretion was also shown to be pulsatile with a pulse interval of 52-54 min. As with GRH, the two analytic techniques used to determine pulse frequency gave comparable results. Although the SRIH frequency was slightly faster than that of GRH, the small number of animals studied, the limited time period $(5 \mathrm{~h})$ of each study, and the interanimal variability does not permit a statistical distinction between the pulse frequencies of the two hormones.

GH secretion, as reflected by measurements in jugular plasma, was also pulsatile with a frequency of one pulse every 58-65 min, intermediate between that of GRH and SRIH. An initial elevation of GH values seen in two of the sheep is believed to reflect a stress response before initiation of sample collection.

The analysis of the interaction between GRH and SRIH also required a novel analytic approach and we initially attempted to compare directional changes in individual hormone concentrations. Although considerable concordance (GRH vs. GH) and discordance (SRIH vs. GH) were found, these values were not statistically distinguishable from those expected on the basis of chance observations alone. When the same analysis was applied to the hormone secretory peaks rather than the individual values, however, a highly significant association was seen between peaks of GRH simultaneous with or immediately preceding both GH peaks and initiation of the GH pulse. This association could explain the majority (62\%) of the GH peaks observed. In contrast, no such association was observed between SRIH troughs and GH peaks or between GRH and SRIH peaks in portal plasma. 
Table I. Relationships among GRH, SRIH, and GH Peaks (Method 1: Detect)

\begin{tabular}{|c|c|c|c|}
\hline Comparison & No./total (\%) & $x^{2}$ & $P$ \\
\hline \multicolumn{4}{|c|}{$\begin{array}{l}\text { GH peak coincident with GRH } \\
\text { peak }\end{array}$} \\
\hline Experimental & $8 / 21(38)$ & & \\
\hline Random & $13 / 84(15)$ & 4.05 & 0.044 \\
\hline \multicolumn{4}{|c|}{$\begin{array}{l}\text { GH peak coincident or one sample } \\
\text { after GRH peak }\end{array}$} \\
\hline Experimental & $13 / 21(62)$ & & \\
\hline Random & $26 / 84(31)$ & 5.63 & 0.018 \\
\hline \multicolumn{4}{|c|}{$\begin{array}{l}\text { GH peak coincident with SRIH } \\
\text { peak }\end{array}$} \\
\hline Experimental & $2 / 29(7)$ & & \\
\hline Random & $22 / 116(19)$ & 1.65 & NS \\
\hline \multicolumn{4}{|c|}{$\begin{array}{l}\text { GH peak coincident or one sample } \\
\text { after SRIH peak }\end{array}$} \\
\hline Experimental & $6 / 29(27)$ & & \\
\hline Random & $44 / 116(38)$ & 2.34 & NS \\
\hline \multicolumn{4}{|c|}{$\begin{array}{l}\text { GRH peak one sample before } \\
\text { SRIH peak }\end{array}$} \\
\hline Experimental & $4 / 21(19)$ & & \\
\hline Random & $13 / 84(15)$ & 0.01 & NS \\
\hline \multicolumn{4}{|c|}{$\begin{array}{l}\text { SRIH peak one sample before } \\
\text { GRH peak }\end{array}$} \\
\hline Experimental & $6 / 29(21)$ & & \\
\hline Random & $18 / 116(16)$ & 0.15 & NS \\
\hline \multicolumn{4}{|l|}{ GH peak coincident with } \\
\hline GRH peak & $8 / 21(38)$ & & \\
\hline SRIH peak & $2 / 27(7)$ & 5.59 & 0.018 \\
\hline \multicolumn{4}{|c|}{$\begin{array}{l}\text { GH peak coincident or one sample } \\
\text { after }\end{array}$} \\
\hline GRH peak & $13 / 21(62)$ & & \\
\hline SRIH peak & $6 / 29(27)$ & 7.12 & 0.008 \\
\hline \multicolumn{4}{|c|}{ GRH peak one sample before } \\
\hline SRIH peak & $4 / 21(19)$ & & \\
\hline \multicolumn{4}{|c|}{ SRIH peak one sample before } \\
\hline GRH peak & $6 / 29(27)$ & 0.05 & NS \\
\hline
\end{tabular}

Experimental data are those from comparisons within individual animals. Random values are those from comparisons of the peaks of one hormone from one sheep with those of the second hormone from the other four sheep.

These findings provide evidence for a primary role of GRH in the pulsatile secretory pattern of $\mathrm{GH}$ secretion. The absence of an observed association between SRIH troughs and GH pulses suggests that SRIH exerts a less important role in initiating GH pulses. However, the possibility exists that the intensity of the GH pulse (i.e., pulse height and duration) are to an extent modified by the level of SRIH at the time of the GRH pulse. Attempts to demonstrate such a relationship in the present study were, however, unsuccessful in part because of the small number of pulses in individual animals and the interanimal variability in hormone levels. The lack of a complete coupling of either GRH or SRIH with GH pulses also raises the possibility of yet another hypothalamic factor that contributes to $\mathrm{GH}$ pulsatility. The nature of this postulated factor, however, remains unknown.
Table II. Relationships among GRH, SRIH, and GH Peaks (Method 2:Three CV Criteria)

\begin{tabular}{lccc}
\hline \multicolumn{1}{c}{ Comparison } & No./total (\%) & $x^{2}$ & $P$ \\
\hline $\begin{array}{l}\text { Initiation of GH peak coincident } \\
\text { with GRH peak }\end{array}$ & & & \\
$\quad \begin{array}{l}\text { Experimental } \\
\text { Random }\end{array}$ & $8 / 29(28)$ & & \\
Initiation of GH peak coincident & $10 / 106(9)$ & 5.02 & 0.025 \\
$\quad \begin{array}{l}\text { or one sample after GRH } \\
\text { peak }\end{array}$ & & & \\
$\begin{array}{l}\text { Experimental } \\
\text { Random }\end{array}$ & $14 / 29(48)$ & & \\
$\begin{array}{l}\text { Initiation of GH peak coincident } \\
\quad \text { with SRIH minimum }\end{array}$ & $23 / 106(22)$ & 6.80 & 0.009 \\
$\quad \begin{array}{l}\text { Experimental } \\
\text { Random }\end{array}$ & $5 / 29(17)$ & & \\
$\begin{array}{l}\text { Initiation of GH peak coincident } \\
\text { with or one sample after }\end{array}$ & $25 / 106(24)$ & 0.23 & NS \\
$\quad$ SRIH minimum & & & \\
$\begin{array}{l}\text { Experimental } \\
\text { Random }\end{array}$ & $10 / 29(34)$ & & \\
\hline
\end{tabular}

Experimental and random as defined in Table I.

Hypoglycemia in the sheep resulted in a decrease in plasma GH levels. Similar findings have been previously reported in the rat (19). Previous studies have suggested a mechanism to explain this response since neuroglycocytopenia induced by 2-deoxyglucose is a potent stimulus for SRIH release by rat hypothalamic fragments in vitro (20). The present results clearly demonstrate that insulin-induced hypoglycemia in sheep stimulates an immediate release of SRIH from the median eminence into the portal system with peak levels nearly double those before injection occurring $20 \mathrm{~min}$ after insulin injection. GH concentrations exhibited a corresponding decrease, which was significant during the second hour after injection. In contrast, GRH concentrations appeared unaltered by the injection of insulin for $90 \mathrm{~min}$, after which a slight increase was observed. This was well beyond the time when SRIH values had returned to preinjection levels. In addition, GRH pulsatility persisted in three of the five animals during the first $\mathbf{9 0} \mathrm{min}$ after insulin administration.

There is considerable indirect evidence that suggests an inhibitory effect of SRIH on GRH secretion $(6,10,21)$. However, the present results indicate either that $(a)$ the increased release of SRIH into the portal circulation in response to insulin-induced hypoglycemia is unaccompanied by a concomitant SRIH release at nerve terminals in the region of GRHcontaining cell bodies, or $(b)$ the concentrations of SRIH required to inhibit GRH release are greater than those occurring in association with the increased levels detected in the portal circulation. The present results also do not permit a distinction to be made between the effects of increased SRIH secretion, the hypoglycemia induced by insulin, or a separate effect of insulin itself on the suppression of $\mathrm{GH}$ secretion. The prolonged suppression, well beyond the time that SRIH levels returned to baseline, suggests that the effect cannot be explained by SRIH alone. At the dose used in the present study, 

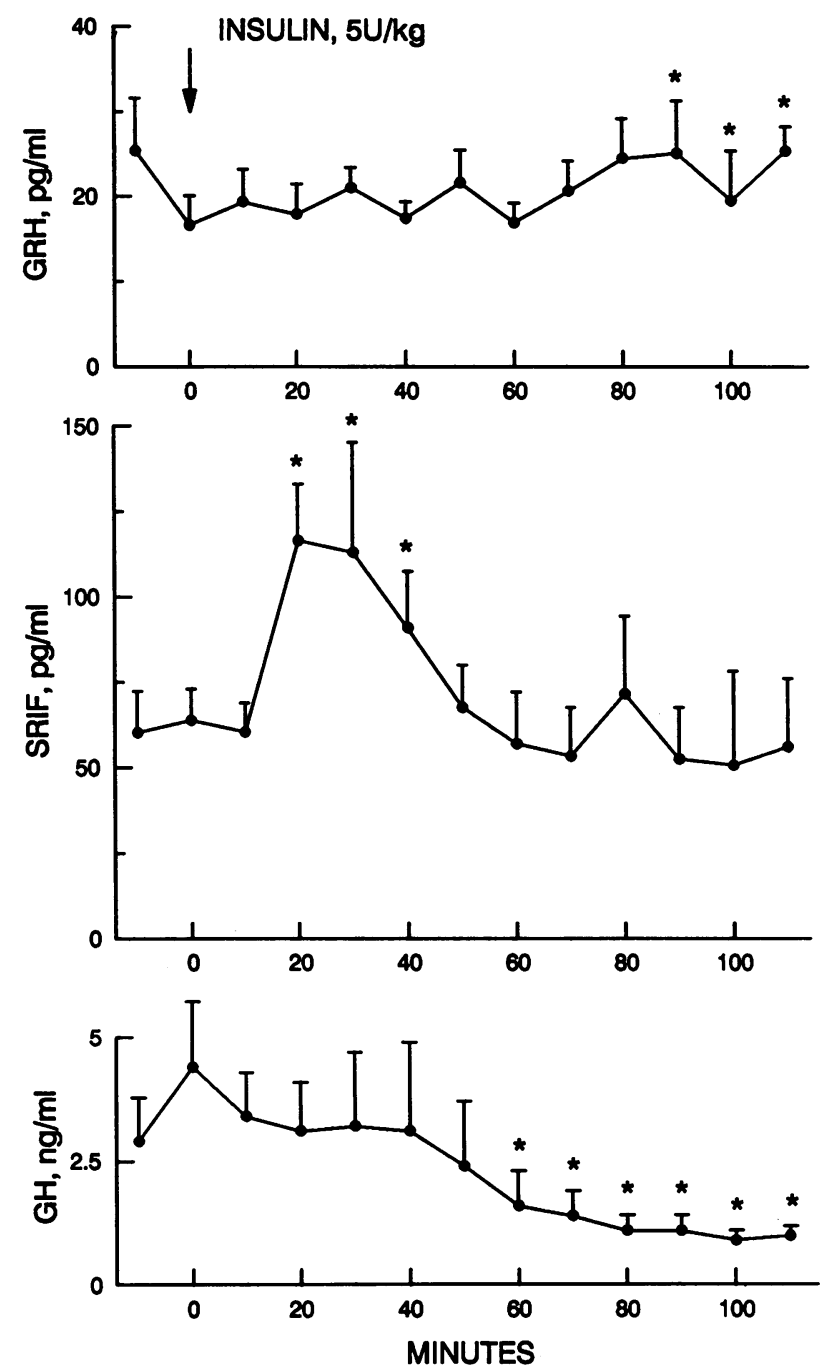

Figure 3. Mean \pm SE of portal plasma GRH and SRIH levels and of jugular plasma GH levels in five unanesthetized ovariectomized ewes in response to insulin hypoglycemia. Asterisks indicate paired differences between the mean value at individual time points and those immediately before injection of insulin with a $P<0.05$.

insulin may have exerted short-term effects through CNS IGF-I receptors to which it binds, though with reduced affinity (22), to stimulate SRIH release (23). In addition, direct effects of insulin on pituitary IGF-I receptors may have contributed to the more prolonged suppression of GH secretion, as has been previously reported $(23,24)$. Moreover, it is possible that the hypoglycemia, in addition to its stimulatory effect on SRIH secretion (20), may have affected the release of another, yet unidentified factor that influences GH secretion.

Finally, our results in the sheep (as in the rat) are in marked contrast to those in humans where insulin-induced hypoglycemia increases circulating GH concentrations (25) and indirect evidence exists for the suppression of SRIH secretion, based on the enhancement by insulin of the GH response to GRH (26). The explanation for this species difference remains unknown.

In summary, the present studies have described pulsatile patterns of GRH and SRIH in hypothalamic-pituitary portal plasma that appear to reflect independent neural rhythmicity. The results indicate a primary role of GRH in determining pulsatile GH secretion, with that of SRIH being less important. Insulin-induced hypoglycemia leads to a rapid and brief increase in hypothalamic SRIH secretion and a more delayed and prolonged decrease in plasma GH levels. The persistence of suppression of GH secretion, however, also implies the participation of other inhibitory factors.

\section{Acknowledgments}

We thank Jane Withrow, Heather Francis, Bruce Doughton, and Paul Weston for their excellent technical assistance and the Director of the Animal Research Institute at Werribee for the use of animal facilities. Materials for the ovine GH assay were provided by the National Institute of Arthritis, Diabetes, and Digestive and Kidney Diseases Hormone Distribution Program.

These studies were supported in part by U. S. Public Health Service grant DK-30667 and the National Health and Medical Research Council of Australia.

\section{References}

1. Frohman, L. A. 1987. Diseases of the anterior pituitary. In Endocrinology and Metabolism. P. A. Felig, J. Baxter, A. E. Broadus, and L. A. Frohman, editors. 2nd ed. McGraw-Hill Book Company, New York. 247-337.

2. Wehrenberg, W. B., P. Brazeau, R. Luben, P. Bohlen, and R. Guillemin. 1982. Inhibition of the pulsatile secretion of growth hormone by monoclonal antibodies to the hypothalamic growth hormone releasing factor (GRF). Endocrinology. 111:2147-2148.

3. Ferland, L., F. Labrie, M. Jobin, A. Arimura, and A. V. Schally. 1976. Physiological role of somatostatin in the control of growth hormone and thyrotropin secretion. Biochem. Biophys. Res. Commun. 68:149-156.

4. Frohman, L. A., T. R. Downs, H. Katakami, and J.-O. Jansson. 1987. The interaction of growth hormone-releasing hormone and somatostatin in the regulation of growth hormone secretion. In Growth Hormone: Basic and Clinical Aspects. O. Isaksson, C. Binder, K. Hall, and B. Hokfelt, editors. Elsevier Science Publishers B. V., Amsterdam. 63-77.

5. Tannenbaum, G. S., and N. Ling. 1984. The interrelationship of growth hormone $(\mathrm{GH})$-releasing factor and somatostatin in generation of the ultradian rhythm of GH secretion. Endocrinology. 115:19521957.

6. Katakami, H., T. R. Downs, and L. A. Frohman. 1988. Inhibitory effect of hypothalamic medial preoptic area somatostatin on growth hormone-releasing factor in the rat. Endocrinology. 123:11031109.

7. Patel, Y. C., and C. B. Srikant. 1986. Somatostatin modulation of adenohypophysial secretion. Annu. Rev. Physiol. 48:551-567.

8. Kashio, Y., K. Chihara, T. Kita, Y. Okimura, M. Sato, S. Kadowaki, and T. Fujita. 1987. Effect of oral glucose administration on plasma growth hormone-releasing hormone (GHRH)-like immunoreactivity levels in normal subjects and patients with idiopathic GH deficiency: evidence that GHRH is released not only from the hypothalamus but also from extrahypothalamic tissue. J. Clin. Endocrinol. Metab. 64:92-97.

9. Plotsky, P. M., and W. Vale. 1985. Patterns of growth hormonereleasing factor and somatostatin secretion into the hypophysial-portal circulation of the rat. Science (Wash. DC). 230:461-463.

10. Clarke, I. J., and J. T. Cummins. 1982. The temporal relationship between gonadotropin releasing hormone (GnRH) and luteinizing hormone (LH) secretion in ovariectomized ewes. Endocrinology. 111:1737-1739. 
11. Clarke, I. J., G. B. Thomas, B. Yao, and J. T. Cummins. 1987. GnRH secretion throughout the ovine estrus cycle. Neuroendocrinology. 46:82-88.

12. Gordon, K., M. B. Renfree, R. V. Short, and I. J. Clarke. 1987. Hypothalamo-pituitary portal blood concentrations of beta-endorphin during suckling in the ewe. J. Reprod. Fertil. 79:397-408.

13. Engler, D., T. Pham, M. J. Fullerton, J. W. Funder, and I. J. Clarke. 1988. Studies of the regulation of the hypothalamic-pituitaryadrenal axis in sheep with hypothalamic-pituitary disconnection. I. Effect of an audiovisual stimulus and insulin-induced hypoglycemia. Neuroendocrinology. 48:551-560.

14. Frohman, L. A., and T. R. Downs. 1986. Measurement of growth hormone-releasing factor: Methods Enzymol. 124:371-389.

15. Kronheim, S., M. Berelowitz, and B. L. Pimstone. 1978. The characterization of somatostatin-like immunoreactivity in human serum. Diabetes. 27:523-529.

16. Williams, T. C., M. Kelijman, W. C. Crelin, T. R. Downs, and L. A. Frohman. 1988. Differential effects of somatostatin and a somatostatin analog, SMS 201-995, on the secretion of growth hormone and thyroid-stimulating hormone in man. J. Clin. Endocrinol. Metab. 88:39-45.

17. Guardabasso, V., G. DeNicolao, M. Rocchetti, and D. Rodbard. 1988. Evaluation of pulse-detection algorithms by computer simulation of hormone secretion. Am. J. Physiol. 255:E775-E784.

18. Oliver, C., R. S. Mical, and J. C. Porter. 1977. Hypothalamicpituitary vasculature: evidence for retrograde blood flow in the pituitary stalk. Endocrinology. 101:598-604.

19. Schalch, D. S., and S. Reichlin. 1968. Stress and growth hor- mone release. In Growth Hormone. A. Pecile and E. E. Muller, editors. Excerpta Medica Foundation, New York. 211-225.

20. Berelowitz, M., D. Dudlak, and L. A. Frohman. 1982. Release of somatostatin-like immunoreactivity from incubated rat hypothalamus and cerebral cortex: effects of glucose and glucoregulatory hormones. J. Clin. Invest. 69:1293-1301.

21. Liposits, Z., I. Merchenthaler, W. K. Paull, and B. Flerko. 1988. Synaptic communication between somatostatinergic axons and growth hormone-releasing factor (GRF) synthesizing neurons in the arcuate nucleus of the rat. Histochemistry. 89:247-252.

22. Rosenfeld, R. G., and R. L. Hintz. 1986. Somatomedin receptors: structure, function, and regulation. In The Receptors. Vol. III P. M. Conn, editor. Academic Press, Orlando, FL. 281-329.

23. Berelowitz, M., M. Szabo, L. A. Frohman, S. Firestone, L. Chu, and R. L. Hintz. 1981. Somatomedin-C mediates growth hormone negative feedback by effects on both the hypothalamus and the pituitary. Science (Wash. DC). 212:1279-1281.

24. Brazeau, P., R. Guillemin, N. Ling, J. Van Wyk, and R. Humbel. 1982. Somatomedin inhibition of the growth hormone secretion stimulated by the hypothalamic factor somatocrinin or the synthetic peptide hpGRF. C. R. Seances Acad. Sci. 295:651-654.

25. Roth, J., S. Glick, R. S. Yalow, and S. A. Berson. 1963. Hypoglycemia: a potent stimulus to secretion of growth hormone. Science (Wash. DC). 140:987-988.

26. Kelijman, M., and L. A. Frohman. 1988. Discordant effects of insulin-hypoglycemia on growth hormone-releasing hormone-stimulated growth hormone and thyrotropin-releasing hormone-stimulated thyrotropin secretion. J. Clin. Endocrinol. Metab. 66:872-875. 